\title{
Du temps compté au temps vécu à l'époque baroque
}

From Counted Time to Lived Time in the Baroque Period

\section{Benito Pelegrín}

\section{(2) OpenEdition}

1 Journals

Édition électronique

URL : https://journals.openedition.org/cher/4279

DOI : $10.4000 /$ cher.4279

ISSN : 2803-5992

Éditeur

Presses universitaires de Strasbourg

\section{Édition imprimée}

Date de publication : 30 novembre 2021

Pagination : 81-96

ISBN : 979-10-344-0095-9

ISSN : 1968-035X

\section{Référence électronique}

Benito Pelegrín, «Du temps compté au temps vécu à l'époque baroque », reCHERches [En ligne], 27 | 2021, mis en ligne le 30 novembre 2021, consulté le 12 janvier 2022. URL : http://

journals.openedition.org/cher/4279; DOI : https://doi.org/10.4000/cher.4279

\section{(c) (i) (2)(2)}

Ce(tte) œuvre est mise à disposition selon les termes de la Licence Creative Commons Attribution Pas d'Utilisation Commerciale - Partage dans les Mêmes Conditions 4.0 International. 


\title{
Du temps compté au temps vécu à l'époque baroque
}

\author{
Benito Pelegrín
}

$\mathrm{L}$ e conflit de générations n'a pas d'époque, métaphorisé et mythifié depuis des temps immémoriaux. Aux archétypes des complexes d'Edipe et d'Électre, désirant et réussissant mort du père et de la mère, répondent aussi les archaïques précédentes figures d'Ouranos, prolifique et monstrueux géniteur, finalement châtré par ses enfants révoltés menés par Chronos qui, à son tour, dévorera les siens. Parricide et matricide ont la symétrique et antithétique pulsion infanticide de l'autre côté du miroir d'un vital et mortel narcissisme affronté où l'un ne peut admettre l'autre.

Conflit éternel entre la vieillesse et la jeunesse que la pandémie du Covid-19 réactualise avec l'urgence de la protection sanitaire donnée aux vulnérables vieux mais avec le corollaire d'un confinement pour tous, vécu par les jeunes comme un injuste sacrifice de leur vitalité, redoublé ensuite par les priorités pour les vaccins, étalonnés en tranches d'âge en étages décroissants, plaçant la jeunesse au dernier. Rancœurs et suspicions réciproques de mise en danger des anciens par l'imprudence et les transgressions sanitaires des jeunes, leurs fêtes clandestines, ou leurs manifs ouvertes, leurs explosions en banlieues, jeunesse d'autant plus menaçante qu'elle déchante au présent avec un futur qui ne chante pas, réactivant le pessimiste «No futur» punk, sauf, pour avenir, l’héritage de la dette à régler: des anciens. Sans compter le passif climatique, futur désastreux, que les jeunes, plus sages que leurs parents, reprochent désormais à l'action industrielle irresponsable et l'inaction coupable de leurs aînés. Et même lors du joyeux mai 68, si les plus indulgents des anciens, non sans envie, ou nostalgie de la leur, soupiraient: "Il faut bien que jeunesse se passe», mettant au rang de dernière inévitable maladie infantile une jeunesse qui ne fait que passer et ne se passe pas, certains, oracles orageux, détenteurs du pouvoir de société toujours

* Benito Pelegrín, agrégé, docteur d'État, professeur émérite des universités, spécialiste du baroque et néobaroque (expert culturel près le Conseil de l'Europe et l'UNESCO). Écrivain, musicologue, dramaturge. 
gérontocratiques, fulminaient: "Il leur faudrait une bonne guerre pour les calmer.»

Cet antagonisme générationnel sans âge est peut-être parlé et pensé pour la première fois par le Baroque, archéologie de notre modernité (Pelegrin , 2008).

\section{Du neuf au Moderne, du moderne au jeune}

La découverte du Nouveau Monde a frappé de caducité l'Europe, devenue l'Ancien Monde: le Baroque, déchirure entre le neuf et l'ancien. Aux découvertes toujours plus grandes de la géographie et de l'astronomie, au trouble de l'indéfinition des limites de la terre et du ciel, s'ajoutent l'éclatement des dogmes politiques, religieux, moraux, Réforme et Contre-Réforme. Pères et repères anciens (papes, princes, idéologies, savoirs traditionnels), sont secoués ou balayés par le vent de la crise.

\section{Du neuf au moderne}

"Il faut être absolument moderne»! Proclame Rimbaud ${ }^{1}$ où le guère plus vieux Musset avait déclaré plus tôt, avec une automnale mélancolie: "Je suis venu trop tard dans un siècle trop vieux.»

En toute époque on s'éprouve moderne dès lors qu'on estime vivre avec son temps, en rupture avec le passé et ses traditions. Le $\mathrm{xx}^{\mathrm{e}}$ siècle, s'est voulu résolument moderne après la Première Guerre mondiale, s'est proclamé postmoderne après la Seconde: on peut à l'évidence rapprocher ces débats et ce vécu d'une modernité avec les polémiques sur le nouveau et le moderne à l'âge baroque.

\section{Siècle nouveau}

Tout siècle qui commence se ressent comme nouveau, l'Art Nouveau ${ }^{2}$ dont se flattait le début du $\mathrm{xx}^{\mathrm{e}}$ triomphant n'est pas une nouveauté. Même par la Contreréforme, le Baroque n'est pas un retour en arrière idéologique; c'est moins une remise en ordre qu'une façon nouvelle de mesurer, de dire et de renommer un ordre nouveau du monde inventé en marchant, qui inaugure sa propre tradition. Ou la nôtre.

Face au «tout est déjà dit» classique fondé sur l'imitation des modèles anciens réputés indépassables, parfaits, donc à jamais achevés, cet art baroque résolument neuf, ce culte de l'invention, de l'originalité, venu d'Italie, proclame,

1 Une saison en enfer, "Adieu», 1873. Le mot «modernité» paraît vers 1830, Baudelaire en théorise la notion entre 1845 et 1863 (Le peintre de la vie moderne). Chateaubriand en revendique l'invention dans ses Mémoires d'Outre-tombe (1848). Pour Stendhal, Racine et Shakespeare (1820), à public nouveau, art nouveau.

2 Art Nouveau pour les Français, les Belges et les Hollandais; Liberty pour les Italiens et les Britanniques, Jugendstil pour les germaniques, Arte joven ou Modernismo pour les Espagnols: ces divers noms associent nouveauté, modernité, jeunesse et liberté dans cet art se ressouvenant du Baroque. 
avec Gracián, que «rien ne s'est dit par rapport à tout qu'il reste à dire. » Même en récupérant et réinterprétant des formes anciennes, le Baroque s'ouvre à la potentialité d'une création toujours renouvelée. À l'image de ce monde nouveau dont les noms renouvellent l'Ancien: Nouvelle-Espagne au sud, NouvelleAngleterre, Nouvelle-France au nord, Terre-Neuve, sans compter ces NouvelleZélande, Nouvelles-Hébrides, et leurs cortèges de Nouvelles provinces ou Nouvelles villes (Pelegrin $2000: 28-30$ ). À cheval entre les $\mathrm{XVI}^{\mathrm{e}}$ et $\mathrm{XVII}^{\mathrm{e}}$ siècles, le Baroque est un art nouveau pour un monde ancien et un univers nouveau.

\section{Nouveau, moderne: Manifestes de la nouveauté}

À l'orée du nouveau siècle, l'église du Gésu de Rome offre le modèle de la nouvelle église; l'atelier des Carrache, signe la mort du maniérisme du $\mathrm{xvI}^{\mathrm{e}}$, ouvre la voie à Caravage, au retour à la couleur et au naturalisme en peinture. À partir de 1600, sur un demi-siècle, on ne compte pas les ouvrages artistiques et scientifiques proclamant leur nouveauté, leur modernité, non sans rivalités et revendications de la primauté: Musiche nove (1600) de Caccini, son Euridice et celle de Peri (1600), Monteverdi (Orfeo, 1607), Arte nuevo de hacer comedias en este tiempo (1609) "Art nouveau pour faire du théâtre pour notre temps» de Lope de Vega. Dans les sciences: De magnete de William Gilbert (1600), "la nouvelle manière de philosopher", Astronomia nova (1609) de Képler; Bacon oppose son Novum organum (1620)3 à celui d'Aristote, la géographie du Père Briet, Paralela geographice veteris et novee (1646), rend caduque celle de Ptolémée. Galilée s'impose comme premier physicien moderne dans son Discorsi intorno a due nuove Scienze (1638).

En changeant de siècle, le seuil du Baroque se considère logiquement nouveau, le proclame et l'on voit naître une nouvelle manière de culture temporelle.

\section{Du culte du nouveau à la culture du temps}

À temps nouveaux, instruments nouveaux qui explorent et mesurent l'univers et le microcosme de l'homme: microscope et lunette astronomique, thermomètre (Galilée), sont inventés à la charnière des deux siècles. J'ajouterai deux emblèmes, sinon deux inventions, deux artéfacts contemporains, connus de toute antiquité, mais qui, grâce à des avancées techniques toutes nouvelles, vont prendre une acuité décisive dans l'auto-connaissance du microcosme qu'est l'homme: le miroir et l'horloge. On s'épargnera le cliché de métaphores baroques rabâchées de la fugacité du temps. Elles n'intéressent ici que comme instruments de l'homme concret dans cet univers baroque de l'obsession numérique (Pelegrin 2009). Soulignons l'idée de mesure du vers du poète Ciro di Pers: "Notre heure se mesure en ombre qui commence.»

3 Citons sa Nouvelle Atlantide (1627). 


\section{Autoportrait}

Difficile, aujourd'hui, d'imaginer un visage sans miroir et un âge sans date, sans heure. Nous devons pourtant l'envisager à l'époque antérieure au Baroque.

Les miroirs anciens étaient en métal poli, l'on en construira jusqu'au XvirI ${ }^{e}$ siècle (Melchior-Bonnet 2014). Au XIII ${ }^{\mathrm{e}}$ siècle, on invente les miroirs en cristal de roche sur une lame métallique, de petites dimensions. Les miroirs convexes postérieurs et de la Renaissance permettaient ces anamorphoses étranges dont témoignent des tableaux et les miroirs déformants des fêtes foraines. Alors, la question que pose la reine médiévale de Blanche-Neige, "Miroir, miroir, dis-moi la vérité? ", se pose bel et bien : le miroir ancien n'était pas fidèle, bien que le sien

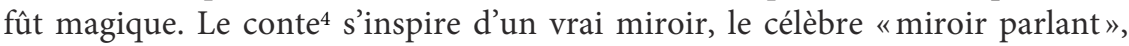
parlant car orné d'une maxime sur la vanité. C'est le miroir plat, de petites dimensions, qui inaugure et facilite le jeu pictural et littéraire de l'autoportrait qui va fleurir au $\mathrm{XVII}^{\mathrm{e}}$ siècle $^{5}$. Des moralistes dénoncent cet instrument qui nourrit la vanité, l'amour-propre, ainsi Monsieur de Moncade qui envisage son époque:

La mode de faire le portrait de soi-même est le plus grand service qu'on ait rendu de nos jours à l'amour-propre. (Moralistes du XVII siècle 1992: 328)

Que dire des selfies d'aujourd'hui? On peut retourner à La Rochefoucauld sa dénonciation de l'amour-propre dans ses Maximes à voir la complaisance, apparemment objective, qu'il met à consulter son miroir pour son fameux Portrait de M.R.D. fait par lui-même:

On m'a dit autrefois que j'avais un peu trop de menton: je viens de me tâter et de me consulter dans le miroir pour savoir ce qui en est.

Rembrandt suit sur son visage le passage troublant du temps, miroir toujours fidèle en peignant un portrait qui change à chaque instant, paradoxe de cette mouvante fixité exprimée par D’Ételan dans le tercet final d'un sonnet au miroir :

Mais toi, peintre brillant, d'un art inimitable,

$\mathrm{Tu}$ fais sans nul effort un ouvrage inconstant

Qui ressemble toujours et n'est jamais semblable.

Image dans l'instant: reflets et réflexions. Ombre et lumière du miroir: lumière qui confronte l'homme à son double, jouant entre réalité et apparence et nous savons avec Lacan, sa fonction dans la prise de conscience de soi par l'enfant. Sujet, j'y vois, objet inversé, ma personne, qui n'est qu'un personnage d'un monde théâtre, tel le regard des autres sur moi? Ou, peut-être, un mirage, espejismo en espagnol, 'jeu de miroir'. Tel celui du miroir de l'amour, «Je t'aime: je voudrais être toi pour me regarder.» Ombre du miroir: cette part de moi

4 Le conte des frères Grimm a un fond historique: le célèbre " miroir parlant» du château de Lohr am Main, haut de 1,60 mètre, produit de la vitrerie de Kurmainz, fondée en 1698.

5 Mlle de Scudéry dans ses romans et Mlle d'Orléans, dans son salon, s'en font une spécialité. 
insaisissable dans le regard de l'autre, témoin de toute cette part de ma vie, que je vis dans la vision d'autrui - qui me demeure à jamais inconnue, que peinture et photo fixent en un instant d'éternité. ${ }^{6}$

\section{Perfectionnement du miroir}

Le perfectionnement technique permet le grand miroir plat au Xvir ${ }^{\mathrm{e}}$ siècle, de plus grandes dimensions. ${ }^{7}$ Le monopole férocement gardé, piraté, échappe à Venise $^{8}$. Une industrie se crée, le miroir devient un objet, un trésor. Des dames se ruinent pour en avoir un seul. Briser un miroir en devient un mauvais présage, perte matérielle mais aussi immatérielle, psychologique.

Le miroir permet la méditation sur l'image et le reflet que sont Les Ménines de Velázquez, sa Vénus au miroir, où l'Amour, qui n'est plus aveugle, voit de face ce qui nous est interdit de dos, sexe de la déesse et visage, dont le reflet de front semble affront à sa beauté dorsale. Le miroir, ouvre son œil dans les Vanités(significativement appelées aussi «Vénus»), allégories picturales de l'inévitable passage du temps. On nomme aussi vanityen anglais les coiffeuses à miroir des dames et l'on connaît les vanity case. Dans les natures mortes, dites également "vanités», le miroir impose sa rhétorique : la spécularité est toujours spéculation et le reflet, réflexion sur soi, le jeu de glaces, qui multiplie les images et les rayons du soleil, est jeu du Je. Parfait exemple, la Galerie des Glaces de Versailles, symboliquement commencée à l'apogée du règne du RoiSoleil, en 1678, quand Versailles devient résidence royale officielle, duplication et multiplication hyperboliques des fastes d'un règne personnel, miroir, admiroir d'un monarque égocentrique et centralisateur.

\section{Horloges}

Les horloges font leur entrée dans les Vanités et dans la poésie morale et scientifique (Pelegrin 2000:283-292), certaines parées d'un miroir. Le sablier du $\mathrm{XVI}^{\mathrm{e}}$ siècle se voit peu à peu supplanté au $\mathrm{XVII}^{\mathrm{e}}$ par les horloges dans les natures mortes 9 . L'horloge a un essor et des progrès contemporains parallèles à ceux de la glace. Paris connaissait dès 1544 une confrérie horlogère, mais ce n'est qu'en 1601 qu'une véritable industrie naît à Genève. La découverte de la loi de

6 Dans certains pays, on peut faire conserver des traces numériques (photos, vidéos, enregistrements, etc.) de soi pour après sa mort: Facebook, face d'éternité?

7 On en suit l'évolution par certains tableaux: dans La femme au miroir du Titien (1515), un homme tend à la femme un miroir du format d'un petit livre, et un autre ovale, plus grand, qui semble convexe, pour qu'elle voie sa chevelure arrière; la Vénus de Véronèse (1585) a un miroir de quelque $30 \mathrm{X} 20 \mathrm{~cm}$, et celle de Velázquez (1647-1651), de 40X30 cm environ.

8 Colbert crée la Manufacture des glaces de Saint-Gobain en 1665 pour éviter les dispendieuses importations des glaces de Venise.

9 Cf. les tableaux d'Antonio de Pereda, Allégorie de la caducité, 1640, Le Rêve du chevalier; David Bailly, Autoportrait avec symboles de vanité, 1651 (montre et sablier); Pieter Claes, Nature morte de vanité (montre). 
l'isochronisme des oscillations du pendule par Galilée permet à Huygens la mise au point en 1658 de la première horloge à pendule, à une seule aiguille. ${ }^{10}$

Même encore très imprécise, elle provoque la naissance d'une florissante industrie des horloges d'appartement ${ }^{11}$ qui viennent orner, tels les précieux miroirs de plus en plus nombreux, les dessus de cheminée, souvent sorte de petit temple à l'antique, à colonnes et chapiteaux encadrant un cadran rond.

La montre, le nom vient de mostrance, ce que l'on montre, dont on fait montre fièrement comme rare objet de luxe, de vanité, dans sa nouveauté, pend à la ceinture des chausses, plus tard dans la poche, est un ressort comique du coup de théâtre dans la comédie à la mode au XVII ${ }^{\mathrm{e}}$ siècle $^{12}$. Gravée, ciselée, niellée, en or, en argent, en cuivre, en laiton, incrustée de pierres précieuses, émaillée, peinte, en tulipe, en carré, à cadran solaire rectangle, en ovale, octogonale, la montre devient ronde et le reste longtemps à partir du milieu du XVII ${ }^{\mathrm{e}}$ siècle.

\section{Idée du temps}

Jusqu'au XVI ${ }^{\mathrm{e}}$ siècle, sans instruments précis pour le mesurer, l'idée, ou plutôt la sensation du temps, était rythmée par des occupations ou préoccupations quotidiennes ou climatologiques: les jours étaient identifiés du nom des saints et de leurs fêtes, non par un chiffre. Le jour, se répartissait en heures canoniales des prières scandées par les cloches, la durée des travaux journaliers, rentrée des bêtes et chants des coqs. On pourrait parler de sentiment climatique du temps selon la saison, et météorologique selon la journée, figuré au mieux par le flou ombreux d'un cadran solaire ne comptant forcément que les heures claires: élasticité et brumeuse mesure du temps. Temps imprécis, dont on savait, la seule certitude, qu'il s'additionne implacablement, sans autre précision que la succession des jours comme le constate avec accablement Macbeth:

Et demain, et demain, et encore demain

Se glisse à petits pas de jour en jour,

Vers l'ultime syllabe du temps du souvenir. $(\mathrm{V}, 5)$

Galilée fera entrer le temps dans la physique en le dotant d'une structure mathématique, une variable de ses lois scientifiques (Klein 2004: 63), sans que, pour autant, on les puisse vérifier, faute d'instruments assez précis de mesure : la seconde se compte encore en pouls, en pulsation: le chronomètre n'est inventé qu'un siècle plus tard (1734)13 (Gassendi 1992: 8-9).

10 Salomon Coster, à La Haye, la fabrique sur les plans de Huygens. Elle faisait le tour du cadran en 24 heures, mais il fallait souvent la remettre à l'heure et était très encombrante. L'aiguille des minutes n'apparaît qu'un peu plus tard au XviI siècle. Le ressort spiral réglant le balancier des montres est inventé par Huygens en 1675.

11 L'Italie semble centre de l'activité horlogère du début du XIV à la fin du XVII ${ }^{\mathrm{e}}$ siècle.

12 Dans Les galanteries du duc d'Ossonne de Mairest (1632), la montre du duc sonne inopinément, II, 3, (didascalie v. 619); dans Le Menteur de Corneille (1645) Dorante se prétend trahi dans la chambre d'une dame par sa montre déréglée sonnant deux fois en un quart d'heure II, 5, v. 632-641. La montre-bracelet est du $\mathrm{xx}^{\mathrm{e}}$ siècle.

13 À Rome la vérification de la loi de Galilée sur la chute des corps échoue faute de 


\section{Vivre avec et dans le temps}

\section{Une autre temporalité?}

L'objet n'existant et ne prenant sens que par la conscience qui le regarde, on tentera une approche psychologique d'horloge et miroir. Certes, avec le surplomb chronologique, nous ne parlons que d'un abstrait possesseur privilégié de ces deux objets exceptionnels et de pointe à l'époque, anticipant leur généralisation chez l'homme d'aujourd'hui.

L'horloge, dont les aiguilles font perpétuellement le tour du cadran, matérialisent désormais pour la vue et pour l'oreille, immuable tic-tac, ce temps toujours égal à lui-même et toujours inégal pour l'homme qui additionne des minutes, des heures, des jours, qui revenant à leur point de départ, s'annulent mécaniquement dans l'éternel retour du cercle des douze heures du cadran, mais s'accumulent pour nous. On le savait par dicton dans la mémoire et la pierre gravée depuis les Romains: toutes les heures blessent et la dernière tue. Voici, maintenant, ces impeccables et implacables aiguilles, ces lancettes, ces petites lances comme les nomment expressivement les Italiens, qui se fichent dans le cœur, qui se plantent dans l'âme en matérialisant pour l'esprit le sentiment tragique d'un temps qui passe et blesse inexorablement à mort notre fragile chair, paradoxale arithmétique: chaque instant de plus est un instant de moins.

\section{De la science à la conscience}

Ces deux artéfacts de la science passent donc à la conscience.

Visage dans le miroir et regard sur l'horloge, c'est sans doute un bouleversement pour l'homme de l'époque baroque. Jusque-là, le temps était lentement rythmé par les saisons, cadencé paresseusement par la sonnerie des cloches, le carillon des «jacquemarts» ${ }^{14}$, les automates, par le cadran solaire variant au gré des soleils et du temps: vague temps sonore ou visuel forcément extérieur qui, maintenant, avec l'aiguille des minutes, calcule ce compte inéluctable à l'intérieur d'une demeure, dans l'intime d'une conscience. Qui peut suivre dans l'image, plus fidèlement rendue par le miroir nouveau, les signes du passage du temps. Le miroir montre donc objectivement l'espoir perdu de notre permanence que chacun peut jauger en se jugeant à son image métamorphosée

pouvoir mesurer avec précision la durée de la chute d'un projectile, faute de savoir compter les secondes. Mersenne (Harmonie universelle) mesure la seconde au battement du pouls d'un homme au repos; «la minute est le temps qu'il faut pour faire 60 pas sans trop se presser. » Le chronomètre de marine de John Harrison (1734) pèse $32.5 \mathrm{~kg}$.

$14 \mathrm{Au}$ Moyen Âge la journée était divisée en six parties appelées horae dont la durée variait selon la saison: la prime (l'aube), la tierce (milieu de la matinée), la sexte (midi), la none (le milieu de l'après-midi), la douzième ou vêpres (le coucher du Soleil), complies. Vers la fin du XIve siècle, la plupart des villes avait abandonné les heures solaires aléatoires et adopté l'horloge mécanique et sa division de la journée en douze parties égales. 
par ce temps dont l'horloge est comptable impeccable: graduation de notre dégradation.

Nous sommes alors dans un autre temps. Ce n'est plus simplement le sentiment tragique de la vie, avec la certitude de la mort brutale au bout, c'est la sensation dramatique mais plus diluée du temps qui passe, doucement instillé, distillé amèrement au quotidien.

Ce temps baroque nouveau, c'est sans doute le temps humain moderne, certes toujours mesuré à l'éternité, symbolisée par la machine infernale de l'horloge de plus en plus précise, qui dit le passage de l'heure pour nous et son indifférence circulaire, mais qui nous fait peut-être moins vivre dans l'angoisse métaphysique du temps que dans la hâte physique pressante et oppressante de l'heure d'un monde mesuré, compté, comptabilisé, compétition avec soi-même des bracelets connectés, vertigineux comptables de pas, de kilomètres, de rythme cardiaque... Folie quantitative qu'inaugurait le Baroque: Friedrich Spee calculait les rythmes vitaux, supputait le nombre de nos respirations journalières, estimait à cinquante ou quatre-vingt-milles les pulsations quotidiennes de notre sang: pour les vouer à Dieu. Aujourd'hui : ici et maintenant.

L'individu des temps modernes, qui commencent avec le Baroque, peut désormais se mesurer à soi avec la cruauté crue du miroir comme il le fera, des siècles après, avec la froide objectivité de la photographie: c'est un défi à sa subjectivité qui ne pouvait avoir jusque-là qu'une conscience imaginaire de soi sans le support concret de l'image précise du miroir. Miroir et horloge, mesurant notre physique et mesurant notre temps, nous confrontent d'abord à nousmêmes. Mais, par la comparaison, ils nous confrontent ou affrontent aux autres.

Talleyrand aura beau dire: "quand je me vois, je me désole, quand je me compare, je me console", on tend à se comparer surtout aux jeunes. Il y a cette vraie ou fausse sérénité de Shakespeare mesurant son âge à celui de son jeune amant:

Mon miroir vainement dira que je suis vieux

Tant que jeunesse et toi vous serez du même âge. (Sonnet XXII)

\section{L'âge à l'époque baroque}

\section{Dictionnaires: âges de l'homme}

Pour définir les âges, en Espagne, le Diccionario de Autoridades (1732) du crépuscule du Baroque coïncide avec celui de son aurore, le Covarrubias (1611), sans différence notable avec les Français.

Derrière les chiffres, la logique symbolique, religieuse: le 7 , le 30 qui scandent les âges de l'homme. Comme celle du monde, la vie de l'homme est divisée en sept âges: de l'enfance à la décrépitude. L' «âge de raison», annos discretionis», est fixé par le droit canon ${ }^{15}$ à 7 ans, l'âge nubile pour le garçon,

15 Concile de Latran IV (1215), qui édicte aussi les décrets du mariage, repris par le 
à quatorze. "La fleur de l'âge", la jeunesse va de 21 à 30 ans, âge où Adam fut chassé du Paradis. Jésus fut crucifié en 30, certains disent à 30 ans, d'autres à 33, pour la symbolique doublement trinitaire. La vieillesse, l'âge décrépit, commence à 60 ans pour les dictionnaires espagnols, le Dictionnaire de Trévoux (entre 1704 et 1771) remet l'âge décrépit à 65 ans que, dans le sien, Furetière (1690), plaçait «au-dessus de 75 ans». Et toujours la fatale charnière: «Entre deux âges, c'est à 30. Ans" [sic]. Et il rappelle: "L'âge de l'homme a esté borné à 120. Ans [sic] en la Genèse Chap. 5 ».

\section{La femme et le temps: littérature et histoire}

On a fait, à la femme, de la beauté, une obligation, qui commence à atteindre les hommes. Le vieillissement passe presque pour une maladie honteuse, un renoncement personnel à rester jeune. On le pardonnait mal à ces favorites entrées dans l'Histoire pour leur jeunesse et beauté, reléguées dès leur précoce automne. Il en va de même pour les hérö̈nes de fiction, exemples français clairement formulés. Jeunesse et beauté, motifs d'amour: cœur des occupations et préoccupations.

Bien sûr, les héros de théâtre et de roman sont jeunes. C'est surligné chez Madame de Lafayette [Pelegrin 2008: 199]. Entre regards et glaces impitoyables de la cour: témoignages féminins pour apprécier à quel miroir se mesurait la jeunesse de la femme aux yeux contemporains.

\section{Entre fiction et réalité}

Le plus long roman de la littérature française (13000 pages), succès grandiose en son temps, Artamène ou le Grand Cyrus (1649-1653), de Georges et Madeleine de Scudéry (1607-1701) - admirable longévité - précieuse donnant le ton dans son célèbre salon, est un document littéraire littéralement précieux et socialement parlant. Long catalogue de précieuses avec leurs pseudonymes transparents et leurs portraits, qui romance les discussions du salon. On y peut jauger le jugement de telle de ses héroïnes sur la jeunesse et la vieillesse des femmes. Ainsi, Amathilde, jeune beauté de dix-sept ans; on lui prédit une vie de cent ans, elle se récrie d'horreur à l'idée de voir dans le même miroir, "conseiller des grâces» des précieuses, des marques du temps car elle met «à vingt-cinq ans» la «vieillesse» d'une femme:

[...] quand je songe à la différence qu'il y a d'une Fille de quinze ans, à une Femme de soixante, j'ay bien moins de peine à me résoudre à mourir à vingt, qu'à aller seulement jusques à cinquante.

Elle ajoute:

Ha si ce malheur m'arrive [...] je casseray tous mes Miroirs, je fuïray autant le monde qu'il me fuïra; et je pense mesme que je ne vous regarderay plus, de peur de me voir dans vos yeux. (de Scudéry 1656: 5553)

Elle exprime un «jeunisme» ravageur contre ses aînées.

Concile de Trente (1545-1563). 
En 1667 un Louis XIV de vingt-neuf ans, après avoir croqué tant de tendrons, fait sa favorite d'une Montespan... déjà de vingt-sept! C'est un choc, à cause de son âge.

À la célèbre courtisane libertine Ninon de Lenclos (1620-1705), morte à quatre-vingt-cinq ans, on prête cette réponse à un jeune abbé impatient de voir combler ses vœux: "Monsieur, pour répondre à vos désirs, je n'attendais que d'avoir fêté mes 80 ans.» Vérité ou légende exceptionnelle à rebours de cette femme de tous les défis.

Madame de La Fayette, a passé l'âge canonique ${ }^{16}$, elle a quarante-quatre ans en publiant son roman La Princesse de Clèves (1678). Histoire à clés, où la cour de Louis XIV est transposée au temps des Valois. L'héroïne, d'" une extrême jeunesse", s'étonne des amours insolites du roi Henri II pour Diane de Poitiers, de vingt ans son aînée (déjà maîtresse de l'ancien roi, François I ${ }^{\text {er}}$ ), devenue sa favorite à l'âge de trente-neuf ans :

"Madame de Clèves, qui était dans cet âge où l'on ne croit pas qu'une femme puisse être aimée quand elle a passé vingt-cinq ans, regardait avec un extrême étonnement l'attachement que le roi avait pour cette duchesse, qui était grandmère, et qui venait de marier sa petite-fille.» (Mme de Lafayette - 2020 :137, 155, 163)

Mais elle-même, dans sa quarantaine, est contradictoirement fâchée de passer pour la maîtresse de La Rochefoucauld - et qu'on l'en croie incapable. Elle s'indigne des jeunes gens qui dédaignent les femmes mûres: «Il semble qu'on leur paraît cent ans dès qu'on est plus vieille qu'eux. » La belle-sœur de la féministe auteure du Grand Cyrus, sceptique sur ces amours prêtées à l'écrivaine comtesse de quarante et un ans avec le duc de La Rochefoucauld de soixante et un, conclut cruellement: "Ils ne sont pas d'âge à faire autre chose ensemble». Plus tard, dans un salon, voyant entrer un très vieux couple, une voix malicieuse murmura ce vers de tragédie: «Et ces deux grands débris se consolaient ensemble.»

En résumé, le bel âge était estimé entre quinze et vingt-cinq ans pour les femmes. ${ }^{17}$ Inégalité biologique, injustice culturelle entre hommes et femmes? Shakespeare vieillissant, exhorte son jeune amant à se reproduire en beauté dans un enfant avant d'atteindre, à quarante ans, la fin de sa jeunesse (Sonnet II).

\section{Vision et aversion des vieux: gérontophobie}

Un homme renonçait «à la vie active vers cinquante ans» (Brighelli 2006: 97), âge de l'incapacité définitive à porter les armes ou à engendrer - à donner la vie ou la mort. Mais l'homme désarmé, donc impuissant, garde la puissance

16 L'âge canonique est de 40 ans minimum pour devenir bonne de curé agréée sinon agréable.

17 La femme de trente ans d'Honoré de Balzac (1829-1842) tente de prouver qu'une femme de cet âge peut encore avoir un attrait sexuel. 
politique, économique. D'où le conflit avec le jeune. Dans son Dictionnaire, au mot vieux, Furetière, donne ce conseil: «Pour vivre long-temps, il faut être vieux de bonne heure, c'est-à-dire, il faut se conserver. ${ }^{18}$

Si l'on devenait vieux plus vite ${ }^{19}$ [Bourdelais-1993 : 19], la vieillesse durait donc d'autant plus, laissant une obscure masse de personnes mûres face à une fragile écume juvénile, non sans conflits.

\section{Épée de Damoclès du mariage}

L'héritage, les parents le conditionnent à l'obéissance des enfants aux mariages arrangés selon leurs propres intérêts politiques et économiques, abus contre le droit canon qui fait du mariage, dès l'âge nubile de douze ans pour les filles et quatorze pour les garçons, le consentement de deux libres volontés sans obligation d'autorisation parentale ${ }^{20}$. Moins libéral, le droit civil, soucieux des intérêts des familles, rogne et limite cette loi religieuse du libre choix fondamental du couple en repoussant la majorité civile d'une fille à vingt-cinq et d'un garçon à vingt-sept ou trente ans, âges très tardifs à l'époque, pour disposer d'eux-mêmes sans être soumis à l'autorité parentale, au risque d'être déshérités. (Pelegrin 2018). Cette violence parentale en violation avec la liberté canonique du mariage des jeunes, est bien le noyau conflictuel entre les générations, attesté par l'Histoire, le ressort essentiel des intrigues en littérature, au théâtre, de Roméo et Juliette aux comédies de Molière, en passant par la comedia espagnole.

L'époque baroque, en façade plus policée, par la politesse et la police, ne s'embarrasse guère de litotes à l'égard des aînés, des barbons. Hors des textes dits «classiques», filtrés par la censure moraliste et la bienséance, ce n'est pas le respect contraint envers les anciens qui domine ${ }^{21}$, c'est l'expression d'une rude gérontophobie, une aversion ou une haine envers le vieux, avare détenteur de la richesse, et de la vieille, anticipation vivante de la mort.

\section{Littérature}

Mademoiselle de Scudéry peut défendre de féministe façon, les appas d'une femme de vingt-cinq ans, toute sa délicatesse n'en manifeste pas moins une répulsion peu charitable envers telle vieille personne qui «avoit sans doute sur le visage tout ce que la vieillesse y peut imprimer de plus horrible.» (de Scudéry: 138)

Le Roman comique de Francion de Sorel narre une jeune et joyeuse partie: des couples s'égayent dans des chambres mais six chevaliers et dames préfèrent "prendre leurs ébats ensemble»: une partouze. En présidente, une «vieille»,

18 Picasso: «Il faut beaucoup de temps pour être jeune».

$19 \mathrm{Au} \mathrm{XVII}^{\mathrm{e}}$ siècle, «le sentiment de vieillesse vient tôt: Mathurin Régnier se dit vieux à 30 ans, et Molière mettra en scène des barbons quadragénaires.

20 Concile de Latran IV, 1215.

21 Significativement, Montaigne salue le respect des anciens «Des Cannibales» (Essais, I, XXXI). 
pour que ce «corps horrible qui ne fait naître que l'effroi », incite sinon excite les jeunes à jouir sans tarder. Dans les ébats nocturnes, le héros enivré, par erreur, étreint et baise la vieille. La vieille, flattée, le veut récompenser d'un bis: le jeune Francion, découvrant avec horreur son erreur, explose d'une violente grossièreté scatologique. Dépité, il débite, explique :

je prenais ta bouche pour un retrait [WC!] des plus sales [...] ayant envie de vomir j'ai voulu [...] ne jeter mes ordures qu'en un lieu dont l'on ne peut accroître l'extrême infection. [...] si j'ai touché à ton corps, c'est que je le prenais pour quelque vieille peau de parchemin que je trouvais bonne à torcher un trou où ton nez ne mérite pas de fleurer. (Beaufort $1980: 50$ et 66)

Le libertin Théophile de Viau prêche l'abandon aux lois de la naturequi impliquent le respect de chaque âge de la vie, sans les mêler, dont se mêlent tyranniquement les vieillards. Il instruit un implacable procès de la vieillesse qui oppose une sagesse convenue aux naturelles "passions humaines» des jeunes. Les conseils d'une vieille à Thisbé irritent le désir impatient de son amant Pyrame qui se lance dans une longue diatribe contre ce «fantôme vivant»,

Ces vieillards dont l'esprit et le corps abattu

Érigent l'impuissance en titre de vertu, $[\ldots]$

Prétendent contre nous forcer l'ordre du temps

Et que nous soyons vieux en l'âge de vingt ans. (Beaufort 1980: 32)

Âge cruel pour les femmes laides ou vieilles, assimilées aux sorcières traquées sans pitié (Daumas 1996: 167). Ou inacceptable image d'une mort toujours emblématisée par une femme: "Portrait vif de la mort, portrait mort de la vie.»

C'est ce qu'écrit de plus aimable Contre une dame, Charles Timoléon de Sigogne tandis qu'Auvray dépeint une nuit d'amour avec une maîtresse trop vieille et maigre sous de macabres couleurs, linceul les draps, cercueil le lit. On pourrait multiplier les textes espagnols, dans le roman picaresque, chez Quevedo, Gracián.

\section{Dictionnaires: les vieux}

L'horreur de la vieillesse, thème littéraire dirions-nous, si cette appréhension de la vie déclinante, au $\mathrm{XVII}^{\mathrm{e}}$ siècle, n'était corroborée, par la certitude des dictionnaires qui en font un normatif constat. César-Pierre Richelet (1679) tranche les tranches d'âge, définit:

vieillard un homme depuis 40 ans jusqu'à 70 . Les vieillards sont d'ordinaire soupçonneux, jaloux, avares, chagrins, causeurs, se plaignent toujours, les vieillards ne sont pas capables d'amitié.

Relative parité de l'homme avec la femme: "vieille de 41 jusqu'à 70 ans. Les vieilles sont fort dégoûtantes. Vieille décrépite, vieille ratatinée, vieille roupieuse ['morveuse']».

Covarrubias, rappelant le bien et le mal que l'on peut dire des vieux, en rappelle une circonstance aggravante si l'on est femme: "Vieja barbuda, de lejos la saludan, aludiendo a que suelen aojar los niños ». (Vieille barbue, de loin on la salue, car elle porte le mauvais œil aux enfants.) 
Image des pères et tuteurs abusifs, des époux imposés aux jeunes filles dans la réalité, les barbons chenus sont les ridicules vaincus, de la Comedia dell'Arte à l'opéra-bouffe en passant par la comédie, exemples superfétatoires. Plus rare, en poésie, la révolte du barbon berné ou rebuté par une jeune belle, tel l'Anglais Robert Herrick à l'épigramme vengeresse À une dame qui lui reproche ses cheveux gris, qui la commet au temps et au jugement implacable du miroir. On connaît bien, grâce à Brassens, du vieux Corneille, les Stances à Marquise, l'actrice, qui lui a préféré Racine; il lui exprime son dépit amoureux sans trop de galanterie, comparant sa fugace beauté à sa durable gloire littéraire. À l'art mineur de l'interprétation, à l'interprète belle et éphémère de ses œuvres éternelles, il oppose ses pouvoirs de Créateur qui, tel un Dieu, a le pouvoir d'immortaliser par ses ouvres qui le mérite à ses yeux:

Vous ne passerez pour belle

Qu'autant que je l'aurai dit.

Bref échantillon du conflit entre la belle et la bête de quarante ans, dont on laisse la conclusion à la piquante réplique que Tristan Bernard prête à la Marquise d'hier pour fermer le bec à Corneille, mise en musique par Brassens.

\section{Dialectique jeune/vieux}

\section{Jeunisme}

Cette haine palpable contre les vieux, cette gérontophobie va de pair avec l'exaltation, le culte de la jeunesse: le jeunisme, sa mode, sa manière d'être, qui gagne même les personnes d'âge mûr. Les exemples français sont innombrables. Mais un phénomène comme le picarisme, le jeune garçon libre courant le monde, au-delà du courant littéraire de ces romans itinérants, est aussi une façon de vie juvénile qui devient aussi une mode chez les gens plus âgés. On peut rapprocher cette façon précaire de vivre et de voyager de celle de la Beat generation des Allen Ginsberg, William S. Burroughs et surtout Jack Kerouac, dont le récit Sur la route (1957) marquera tant la jeunesse beatnik et hippy des années 60 , débouchant sur les révoltes de 68 qui sont vraiment des conflits entre les générations, entre jeunes et vieux, en France, significativement, contre le Général de Gaulle contraint à la fuite et à la démission.

\section{Société patriarcale, gérontocraties}

Même avec une espérance de vie plus basse que la nôtre, la vérité statistique abstraite fausse une réalité plus complexe. La grande longévité n'est pas rare. Des contemporains s'en étonnent même:

On serait tenté de se croire rapproché du temps des Patriarches ", ces légendaires et testamentaires vieillards, s'émerveille l'abbé Lacroix. Un recensement rétrospectif, milieu du XvirI siècle, Almanach des centenaires anciens et modernes en dresse un inventaire en dix volumes. (Bourdelais 1993: 31) 
Ainsi, une jeunesse impatiente d'accéder aux charges, aux héritages, a pour obstacle des anciens installés dans la durée et le pouvoir. Les sociétés baroques sont patriarcales. L'état fonctionne comme une famille et la famille, comme un état, autoritaires tous deux. Au centre, à la tête de l'un, le Roi, et de l'autre, le Père. Au-dessus de tous, Dieu le Père. Le Père, mari au centre de la famille. Le mariage est au mari ce que le serment d'hommage est au suzerain : le contrat qui lui livre le vassal ou la femme, avec la garantie de la religion, d'une monarchie et d'une famille de droit divin. Même si le monarque accède jeune au pouvoir, il est gouverné par de plus vieux (Mazarin, Anne d'Autriche, régents, favoris).

\section{Conflit œdipien}

Dans l'âge du théâtre qu'est l'époque baroque, il est le fondement même des intrigues, le triomphe du jeune couple sur le père tyrannique ou le riche barbon amoureux pour la comédie. Pour le drame, il suffit de citer Don Juan, le Roi Lear, le Cid. Les nombreuses versions d'Edipe attestent peut-être quelque chose de ce conflit de l'ombre porté aux lumières de la scène, de Corneille à Voltaire qui fait dire au sien:

$\mathrm{O}$ ciel ! et quel est donc l'excès de ma misère

Si le trépas des miens me devient nécessaire?

$\mathrm{Si}$, trouvant dans leur perte un bonheur odieux,

Pour moi la mort d'un père est un bienfait des dieux? $(\mathrm{V}, 2)$

Mais, entre histoire et fiction, celle du conflit entre Philippe II et son fils don Carlos, dont le nœud serait la reine Isabelle de Valois, fiancée du fils mais épousée par le père, montre que la fable, le roman de Saint-Réal (1672) et les pièces d'Otway (1676) et de Schiller (1787), reposant sur l'œdipe freudien, est plus forte que la vérité historique.

Furetière, l'auteur du dictionnaire, dans Le Roman bourgeois (1666), peint un père qui se lamente sur la désobéissance de ses enfants ingrats. Mais ingratitude légitime pour Cyrano. Il ironise sur la dette et le respect qu'on nous dresse à avoir pour les parents qui, en nous engendrant, sans nous consulter, n'ont fait que sacrifier moins au devoir de la nature qu'à leur volupté:

marchez sur le ventre du père qui vous engendra, trépignez sur le sein de la mère qui vous conçut, car d'imaginer que ce lâche respect que des parents vicieux ont arraché à votre faiblesse soit tellement agréable au Ciel [...] je n’y vois guère d'apparence. (Bourdelais 1993: 64)

Au-delà de la fiction, fait historique peut-être significatif: en 1679 éclate et en 1682 on étouffe l'Affaire des poisons. On découvre qu'aux parents riches qui s'attardaient trop en cette terre, les impatients héritiers accéléraient le passage de vie à trépas grâce à de la "poudre de succession », délicat euphémisme pour l'arsenic.

\section{Rousseau, censeur du jeunisme}

Il résume bien la question au crépuscule du Baroque. Lettre sur les spectacles (1758): il condamne théâtre et opéra pour leur immoralité de mettre en scène 
le pouvoir des femmes et le triomphe des jeunes sur les vieux, renversement pervers de l'ordre de la nature. Long réquisitoire contre cette prééminence de la jeunesse dans la société, l'affrontement toujours latent, entre vieillards - s'ils ne se sont écrasés - et jeunes. Il dénonce le manque de respect qu’inspirent « une tête chenue et des cheveux blancs» à "une impudente jeunesse, tandis que les anciens, craintifs et modestes, ou n'osent ouvrir la bouche, ou sont à peine écoutés. » Et pire, pour n'être pas marginalisés, ils préfèrent le ridicule de suivre la mode des jeunes et se «rendre méprisables [...] pour prendre indécemment la parure et les manières de la jeunesse.»

Rousseau appelait de ses vœux un gouvernement des Sages, des Anciens. Même si elle lui doit tant, il ne sera pas exaucé par la proche Révolution, sans doute révolte extrême d'une nation de jeunes ${ }^{22}$ contre un pouvoir monarchique décrépit. Le despotisme du nouveau - symbolique - patriarche Napoléon et son Code Civil seront acceptés et salués parce qu'il restaure l'autorité parentale, la "piété filiale», mises à mal par l'individualisme exacerbé et la licence juvéniles de la Révolution (de Broc 1895: 81-82).

\section{Bibliographie}

Beaufort, J. et Beaufort C., 1980, Le dix-septième siècle, textes littéraires français, Paris, Ed. 19/18.

Bourdelais, P., 1993, Le Nouvel âge de la vieillesse, Paris, Odile Jacob, Histoire:

Brighelli, J., 2006, Le Jeu politique, La Rochelle, Sulliver.

Broc (de), H., [1895] 2015, La vie en France sous Napoléon, FE éditions, collection Brévan.

Daumas, M., 1996, La tendresse amoureuse, XVI ${ }^{\mathrm{e}} \mathrm{XVIII}{ }^{\mathrm{e}}$ siècles, Paris, Librairie académique Perrin, Pluriel.

Gassendi, P., 1992, Initiation à la théorie de la musique, de Gaston Guieu, Edisud. Klein, E., 2004, Les tactiques de Chronos, Paris, Champs Flammarion.

Mme de Lafayette, 2020, La Princesse de Clèves, Paris, Folio Classique nº 3381.

Moralistes du XVII siècle, 1992, Paris, Bouquins, Robert Laffont.

Melchior-Bonnet, S., 2014, Historia del Espejo, Buenos Aires, Edhasa.

Pelegrin, B, 2000, Figurations de l'infini, Paris, Seuil.

Pelegrin, B, 2008, D’un temps d'incertitude, Sulliver.

Pelegrin, B, 2009, «Folie quantitative et aliénation du sujet à l'époque baroque», in Quelle liberté pour le sujet à l'époque de la folie quantitative?, L'Impensé contemporain.

22 Plus peuplée à elle seule que toute l'Europe, la France est « un monstre démographique » dont plus de la moitié des 28 millions d'habitants a moins de 25 ans en 1790. Nombre de grandes figures de la Révolution sont des trentenaires, Saint-Just, le plus jeune, guillotiné à 26 ans. 
Pelegrin, B, (sous presse) «Violencia parental y violación canónica: el matrimonio», in Ier Congreso Internacional de Historia y Pensamiento Moderno: Barroco, crisis, pliegue, Madrid, Universidad complutense, $12 / 12 / 2018$.

Scudéry (de), M., Le Grand Cyrus, partie 8, livre 2, <http://www.artamene.org/ index.php?partie $=8>$, version de 1656, Paris, Courbé. 